\title{
Attribution Mechanisms for Ancillary Service Costs Induced by Variability in Power Delivery
}

\author{
Francesca Bona, Nicolas Gast, Jean-Yves Le Boudec, Pierre Pinson, Senior Member, IEEE, Dan-Cristian Tomozei
}

\begin{abstract}
The increased penetration of renewable energy sources in existing power systems has led to necessary developments in electricity market mechanisms. Most importantly, renewable energy generation is increasingly made accountable for deviations between scheduled and actual energy generation. However, there is no mechanism to enforce accountability for the additional costs induced by power fluctuations. These costs are socialized and eventually supported by electricity customers. We propose some metrics for assessing the contribution of all market participants to power regulation needs, as well as an attribution mechanism for fairly redistributing related power regulation costs. We discuss the effect of various metrics used by the attribution mechanisms, and we illustrate, in a game-theoretical framework, their consequences on the strategic behavior of market participants. We also illustrate, by using the case of Western Denmark, how these mechanisms may affect revenues and the various market participants.
\end{abstract}

Index Terms-Electricity markets, ancillary services, power fluctuations, attribution mechanisms.

\section{INTRODUCTION}

$\mathbf{T}$ HE necessary constant balance between the generation and consumption of electricity is challenged by recent developments in power systems. Mainly, we think of the rapid increase in the penetration of renewable energy sources, mostly wind and solar power, with their inherent fluctuations in power generation over a broad range of temporal and spatial scales [1]. Also, requiring electric loads to become more active could actually induce new types of fluctuations in their consumption patterns [2]. Accommodating the resulting net load fluctuations in a liberalized electricity market environment is one of the current challenges to be faced by system operators. Considering operational time-scales, ancillary service procurement is the key mechanism for ensuring access to the resources necessary to guarantee a reliable and economic operation of power systems. Such services can be split into power and energy related categories: primary reserves act instantly and focus on power only; tertiary reserves focus on energy only; and secondary reserves comprise a mix of both, by relieving primary reserves while acting as a transition before

Manuscript received ??, 2015; revised ??, 2016.

F. Bona and P. Pinson are with the Technical University of Denmark, Department of Electrical Engineering, Kgs. Lyngby, Denmark (email: ppin@dtu.dk).

N. Gast is with INRIA, MESCAL team, Grenoble, France (email: nicolas.gast@inria.fr).

J.-Y. Le Boudec is with EPFL, IC, Lausanne, Switzerland (email: jeanyves.leboudec@eplf.ch.

D.-C. Tomozei is with Cisco, email: dtomozei@ cisco.com.

F. Bona and P. Pinson are partly supported by the Danish Innovation Fund through the projects '5s' - Future Electricity Markets (12-132636/DSF) and CITIES (DSF-1305-00027B). Nicolas Gast was partially supported by the European project Quanticol 600708. full activation of tertiary reserves. A recent overview of various reserves and their purposes in power system operations can be found in [3]. The need to redesign ancillary service markets in a new context of high renewable energy penetration is receiving renewed attention, see e.g. [4], [5], while optimal market design with increased renewable energy generation is an open question [6], [7].

Wholesale electricity markets were proposed to make the operation of power systems more economic and efficient. A key concept is balance responsibility: both power suppliers and consumers are deemed accountable for deviations between their scheduled and actual production (resp., consumption). Renewable energy generation, with its limited predictability, is increasingly required to take part in such mechanisms to be financially responsible for the energy regulation needs it induces. However, balance responsibility is enforced for energy only (through the balancing market), with energy imbalances calculated periodically at a time-unit granularity that depends on the market/power-system (e.g., hourly in the Scandinavian Nord Pool and every 15 minutes in the Dutch APX). In contrast, the costs of power-related regulation services are socialized and nearly always supported by the demand side (see for instance the ENTSO-E overview in [8]). This can be seen as fair if such costs originate from demandside fluctuations only, or if such costs are low and it is not possible to attribute them to the various market actors. We argue here that these costs are non-negligible, while it may be possible to assess the contribution of the various actors of the system to these overall regulation costs. Somehow, current market structures implicitly assume that all participants on the supply side deliver energy at a constant power level during each time-unit. In future power-systems where the need for increased power-related services might substantially originate from the production side, power suppliers should be made accountable as in the case of energy-related regulation.

We propose and analyze some attribution mechanisms for the costs induced by power-related regulation needs. These readily complement existing mechanisms for energy-related regulation. The concept of energy-neutral power profiles enables us to separate the needs of energy- and power-related regulation. Next, the contribution of every actor to the overall power regulation cost is determined based on individual versus overall fluctuations over a market time interval. We investigate, in a game-theoretical framework, the theoretical properties of these mechanisms and we use simulation to illustrate these concepts based on a theoretical model and the realistic test case of Western Denmark, already largely penetrated by wind power generation. We employ German data to assess the costs of power-related regulation and to build representative supply 
curves for power-related system services. Although socializing these costs might have been a natural option so far, we believe it is important to see whether better mechanisms could reveal and share these costs among the various actors of the power system. These new mechanisms could also better support new business cases, e.g., storage and demand response, to support renewable energy integration [9].

The paper is organized as follows. In Section II we assess today's costs of power-related regulation services, based on the test cases of Germany and Western Denmark. We describe our market framework in Section III, complementing marketparticipants' revenues with a component reflecting the powerregulation costs they induce. In Section IV we provide insights into the properties of our attribution mechanisms. We illustrate the concept and analyze the resulting revenues for market participants, using a toy model example in Section $\mathrm{V}$ and the more realistic test-case of Western Denmark in Section VI. We present our concluding remarks and perspectives in Section VII.

\section{EMPiricAl EVIDENCE to SupPort BetTer Accounting For the Cost of Power Variability}

In order to estimate the costs induced by power-related regulation and to quantify their importance in current powersystems, we carried out a country-wide comparison between regulation costs linked to power fluctuations and those related to energy imbalances. The comparison is based on the ratio between costs associated with power-related and energy-related regulation services. The former costs stem from the reservation of primary reserves whereas the latter cover energy-balancing costs through the activation of secondary and tertiary reserves. Note that secondary reserves can also contribute to some form of power-related regulation, by relieving primary reserves after a certain period. The related costs, however, are not made public (and are perhaps not even calculated). Hence, they are disregarded here. A direct consequence is that the ratio values calculated here are a lower-bound estimate on the true ratio between power and energy-related regulation costs. The analysis covers an entire year of operation (2014) and two different European control areas, Western Denmark and Germany. In the former case, data with an hourly resolution can be readily downloaded from the website of the Transmission System Operator (Energinet.dk) of Denmark. The distribution of the ratio between power- and energy-related regulation costs is depicted in Figure 1, based on the 8760 values for 2014. This distribution has a long tail but also a high concentration close to zero, which indicates that, in most cases, power-related regulation is substantially less expensive than energy-related regulation; though in few case the opposite is true, with much higher power-related regulation costs. The central 50\% range of ratio values, given by $1^{\text {st }}$ and $3^{\text {rd }}$ quartiles in Figure 1 , is between 6\% and 29\%. For 2014 in Western Denmark, the overall cost induced by power-related services was $9.9 \%$ of that for energy-related regulation services.

Similarly for the case of Germany, data for the procurement and usage of ancillary services over the same year was retrieved from the internet platform for control reserve

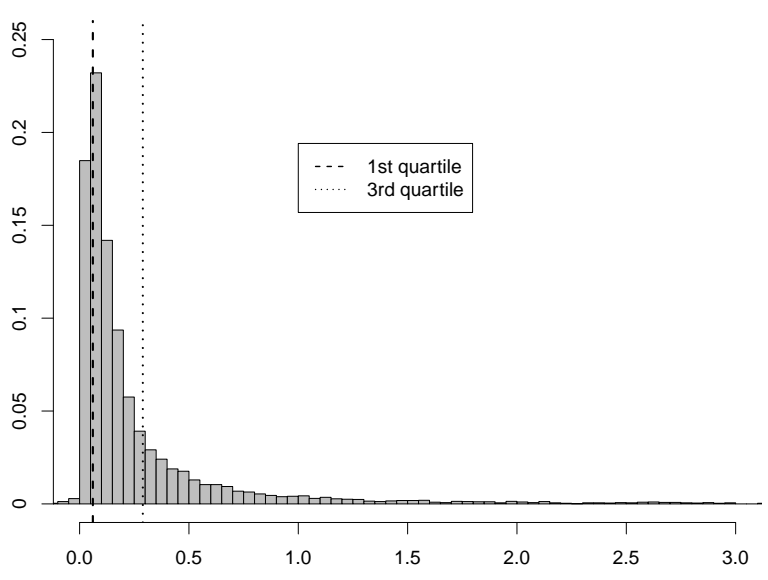

Fig. 1. Histogram of the ratio between costs induced by power-related and energy-related regulation services in Western Denmark in 2014.

tendering (www.regelleistung.net) of the four German TSOs. The overall cost induced by power-related services was $18.1 \%$ of that for energy-related regulation services. For these two power-systems with significant renewable energy generation, it appears that the costs induced by power fluctuations (which could come from both supply and demand sides) are not negligible. Also, the ratio of these costs highly varies throughout the year, depending on weather conditions, operating points of the power systems, etc.

\section{MARKET FRAMEWORK}

\section{A. Power Profiles and Energy-Neutral Power Profiles}

Let us consider a given market participant $i$, who does not participate in the regulation services; this participant can be on the side of supply, demand, or both. It is intuitively expected that the market participants of interest here are those that induce power-regulation needs owing to fluctuations in their power delivery or consumption profiles. Regulation resources are not concerned by the attribution mechanisms described in the following, as they are those who originally provide regulation services.

The power production of participant $i$ is denoted by $q_{i}(t)$, where $t$ denotes time. In the case of a renewable energy generator, this power profile would be a direct function of the weather. Different forms of control (e.g., direct control of generators) allow altering $q_{i}(t)$ and obtain the power profile $p_{i}(t)$ eventually delivered to the market and power system. The absence of control translates to $p_{i}(t)=q_{i}(t)$. We follow the convention that positive power means production whereas negative power means consumption.

We can think of a power profile as a continuous function over a market time unit $\mathcal{T}$, the duration of which is denoted by $T$. In practice, power profiles would be obtained based on a (most likely uniform) sampling of $p$. This would yield the sample power profile $\mathbf{p}_{i}=\left[\begin{array}{llll}p_{i, t_{1}} & p_{i, t_{2}} & \ldots & p_{i, t_{n}}\end{array}\right]^{\top}$ of actor $i$, as a discretized version of $p_{i}$ for a number $n$ of measurement times $t_{j}, j=1, \ldots, n$. To simplify notations in the following, 
we ignore the distinction between continuous and sampled versions of power profiles.

The energy generated by participant $i$ over $\mathcal{T}$ is

$$
E_{i}=\int_{\mathcal{T}} p_{i}(t) d t
$$

Energy imbalances are already settled through existing market mechanisms, thus separating energy and power related aspects. This naturally leads to the definition of the energy-neutral power profile $\tilde{p}_{i}$ of market participant $i$, which we define as its power profile minus its mean over that time-unit, i.e.,

$$
\tilde{p}_{i}(t)=p_{i}(t)-\frac{E_{i}}{T} .
$$

Consequently, power delivery can be split into an energy part $E_{i} / T$ and an energy-neutral power profile $\tilde{p}_{i}$. In practice, when sampling power production, an energy-neutral power profile can be obtained a posteriori at the end of $\mathcal{T}$.

\section{B. Extending Current Market Settlement to Account for Power Profiles}

Consider a market time-unit $\mathcal{T}$, over which market participants have received their energy production and consumption schedules after day-ahead market clearing. Typically in Europe, $\mathcal{T}$ corresponds to $T=1$ hour, though this is expected to shorten in future market designs to better accommodate renewables and pro-active demand. As of now, a market participant $i$ on the supply side has a revenue related to energy only, more precisely its scheduled energy generation $E_{i}^{\mathrm{f}}$ is to be remunerated at the spot price $\pi^{\mathrm{s}}$, whereas imbalances $\left(E_{i}-E_{i}^{\mathrm{f}}\right)$ will be be eventually settled through the balancing market at price $\pi^{\mathrm{b}}$. This yields the overall revenue $R_{i}$ as

$$
R_{i}=\pi^{\mathrm{s}} E_{i}^{\mathrm{f}}+\pi^{\mathrm{b}}\left(E_{i}-E_{i}^{\mathrm{f}}\right) .
$$

In the above, $\pi^{\mathrm{s}}$ is the result of the day-ahead market clearing and $\pi^{\mathrm{b}}$ is the result of the balancing-market clearing, under one-price or two-price settlement approaches. For an extensive discussion on these aspects, the reader is referred to [10]. The market functioning on the demand side is similar, except that revenues become payments.

In this current setup, the market does not give consideration to the power profile that allows for such energy delivery $E_{i}$ over $\mathcal{T}$. Our proposal is to add a new component of the marketrelated revenue that reflects the variability in power-delivery and the consequent need for ancillary services. The revenue of a supplier in the market is hence generalized to

$$
R_{i}=\pi^{\mathrm{s}} E_{i}^{\mathrm{f}}+\pi^{\mathrm{b}}\left(E_{i}-E_{i}^{\mathrm{f}}\right)+R_{i}^{p}
$$

where $R_{i}^{p}$ is the part of the revenue linked to the energy neutral power-delivery profile $\tilde{p}_{i}$. It is intuitively expected to be negative for renewable energy generators, as it penalizes them for fluctuations in their power delivery. Asking a renewable power producer to be accountable for their power-delivery profiles does not go against an overall goal of integrating renewable energies in current power-systems, similarly to the case of energy balancing costs $\left(\pi^{\mathrm{b}}\left(E_{i}-E_{i}^{\mathrm{f}}\right)\right.$ in (4)). We will show and discuss in the following that similar approaches of one-price and two-price settlements can be applied, depending on the mechanisms used to obtain $R_{i}^{p}$. Note that applying controls on original power generation $q_{i}(t)$ to improve the power delivery $p_{i}(t)$ does not affect the day-ahead revenue $\pi^{\mathrm{s}} E_{i}^{\mathrm{f}}$, whereas it potentially affects the revenue from both the balancing market $\pi^{\mathrm{b}}\left(E_{i}-E_{i}^{\mathrm{f}}\right)$ and power delivery $R_{i}^{p}$.

For justification, let us discuss here a natural decomposition of regulation costs in terms of power and energy. First, we define the cost of regulation over a market time-unit $\mathcal{T}$ as the integral of a function $f$ of the total power of non-regulating participants' mismatch at time $t$,

$$
\text { total regulation cost }=\int_{\mathcal{T}} f\left(\sum_{i} p_{i}(t)\right) d t .
$$

This regulation cost includes all costs necessary to ensure safe operation, including power balance and loss compensation. The cost function $f$ is most often considered as quadratic or close to that (see, e.g., [11]). After expanding the expression of $f$ around the total mean power $\sum_{i} \frac{E_{i}}{T}$, the right-hand side in (5) becomes

$$
\int_{\mathcal{T}}\left[f\left(\frac{E_{\mathrm{tot}}}{T}\right)+f^{\prime}\left(\frac{E_{\mathrm{tot}}}{T}\right) \tilde{p}_{\mathrm{tot}}(t)+a\left(\tilde{p}_{\mathrm{tot}}(t)\right)^{2}\right] d t
$$

where $\tilde{p}_{\text {tot }}(t)=\sum_{i} \tilde{p}_{i}(t), E_{\text {tot }}=\sum_{i} E_{i}$ and $a=\frac{1}{2} f^{\prime \prime}\left(\frac{E_{\text {tot }}}{T}\right)$. Following the definition of the energy-neutral power profile in (2), $\int_{\mathcal{T}} p_{\text {tot }}(t)=0$. This gives the decomposition

$$
\text { total regulation cost } \approx R^{e}+R^{p}
$$

$$
\text { with } \quad R^{e}=T f\left(\frac{E_{\text {tot }}}{T}\right), \quad R^{p}=a \int_{\mathcal{T}}\left(\tilde{p}_{\text {tot }}(t)\right)^{2} d t .
$$

Hence the total regulation-cost decomposes into a sum of two terms: one that depends on only the total energy produced by all players and one that integrates the square of the instantaneous power mismatch. The first term is compensated by the balancing market and the ancillary services based only on energy, the second term should be compensated by a mechanism inline with our proposal, focused on variability in power delivery (or consumption). Note that in our proposal, loads and as distributed generation should be accountable for the costs induced by power variability.

There is an interaction between a mechanism that attributes the costs of power profiles, as discussed here, and penalties for energy imbalances. Indeed, in energy markets, a participant that deviates from her dispatch plan has to pay for the deviation and incurs a penalty. Such deviations are measured at the energy timescale, say every 15 minutes. It follows that a participant might have an incentive to mitigate a deviation at the expense of increased variability. To illustrate, consider a market participant that sold 100 units of energy at a price $\pi$, and is able to deliver only 80 units during a time-slot of 15 minutes. Assume the up-regulation price is of $1.5 \pi$. This participant received $100 \pi$ from the day-ahead market and has to buy back $(100-80)$ units when settlement occurs, at a price of $1.5 \pi$, for a total revenue of $70 \pi$. Instead of paying the up-regulation price, this participant might find it more advantageous to produce the missing energy on local additional generators, as long at the generation cost is less than the up-regulation price. For example, after observing during 
10 minutes that her plant is delivering much less power than planned, she could decide to generate additional energy for a duration of 5 minutes, so that the total delivered energy matches the dispatch plan. If she manages to do so, she will deliver the contracted energy, but with large variability. This variability induces costs that will be divided amongst consumers. With our proposal, the cost of variability is made explicit; every participant will have to account for it when preparing dispatch plans and deciding about real-time power controls.

\section{Attribution Mechanisms And their Properties}

We propose and analyze here a number of mechanisms for attributing the costs induced by power fluctuations.

\section{A. Metrics for Attribution of Power-regulation Costs}

After regulation costs $R^{p}$ are known, i.e., after operating over the market time unit $\mathcal{T}$, our proposal is to distribute them among the various actors of the power system (on both supply and demand side), proportionally to a given metric $d_{i}$, defined hereafter, and that depends on the energy-neutral profile $\tilde{p}_{i}$ of actor $i$. The fraction of the power-regulation costs attributed to actor $i$ is then given by

$$
R_{i}^{p}=\frac{d_{i}}{\sum_{j} d_{j}} R^{p}
$$

Various forms of metrics can be defined depending upon the objective of interest. In the following, we focus on metrics that relate to power capacity, integrated mismatch, mileage, and contribution to the total power profile. This last metric should be viewed as different from the others, as it requires comparing individual and total power profiles, whereas the first three are defined based on only individual profiles. In general such metrics are in line with recent developments in regulation markets, where consideration is given to the form of power profiles that will be used for providing regulation, see [12] and references therein.

a) Power Capacity Metric: Our first metric focuses on the capacity required to compensate fluctuations and therefore relates to the $L_{\infty}$ norm of the energy-neutral power profile. Such a metric equally considers positive and negative fluctuations by only focusing on a maximum deviation from the constant power profile. Formally, for an energy-neutral power profile $\tilde{p}_{i}$, related to market participant $i$, the power capacity metric $d_{i}^{\mathrm{pc}}$ is defined as

$$
d_{i}^{\mathrm{pc}}=\left\|\tilde{p}_{i}\right\|_{\infty}=\max _{t \in \mathcal{T}}\left|\tilde{p}_{i}(t)\right|
$$

For a robust estimate $d_{\mathrm{c}, i}$, instead of looking at the maximum in (10), we would prefer to estimate a quantile with nominal level close to 1 from the distribution of the sampled version of $\left|\tilde{p}_{i}\right|$.

b) Integrated Mismatch Metric: Contrary to capacity, which relates to some extreme characteristics of $\tilde{p}_{i}$, with this metric we consider that the integrated mismatch is the quantity of interest, thus relating to a $L_{k}$ norm $(k=1,2)$ of these profiles. Formally, for an energy-neutral power profile $\tilde{p}_{i}$, related to market participant $i$, the integrated mismatch metric of order $k, d_{i}^{\mathrm{im} k}$, is defined as

$$
d_{i}^{\mathrm{im} k}=\left\|\tilde{p}_{i}\right\|_{k}=\left(\int_{\mathcal{T}}\left|\tilde{p}_{i}(t)\right|^{k} d t\right)^{\frac{1}{k}} .
$$

c) Mileage Metric: If, in addition to capacity and integrated mismatch, some of the dynamic properties of energyneutral profiles are of importance, mileage-type metrics can be introduced, related to the $L_{k}(k=1,2)$ norm of the $h$ order derivative of $\tilde{p}_{i}$. In practice, it might be most relevant to stick to the case for which $k=1$ and $h=1$. Hence, for an energy-neutral power profile $\tilde{p}_{i}$, related to market participant $i$, the mileage metric $d_{i}^{\mathrm{m}}$ is defined as

$$
d_{i}^{\mathrm{m}}=\left\|\tilde{p}_{i}^{\prime}\right\|_{1}=\int_{\mathcal{T}}\left|\frac{d \tilde{p}_{i}}{d t}(t)\right| d t .
$$

d) Contribution Metric: In contrast to the previous three metrics, emphasis can be placed on the respective contribution of each market participant to the total power profile. For a given market time-unit, the total energy-neutral profile was introduced in (6). The contribution metric of market participant $i$ depends on both the energy-neutral profile of this participant and total energy-neutral profile. More precisely, for an energyneutral power profile $\tilde{p}_{i}$, related to market participant $i$, its contribution metric $d_{i}^{\mathrm{c}}$ to the total power profile $\tilde{p}_{\text {tot }}$ is defined as

$$
d_{i}^{\mathrm{c}}=\int_{\mathcal{T}} \tilde{p}_{i}(t) \tilde{p}_{\mathrm{tot}}(t) d t .
$$

i.e. $d_{i}^{\mathrm{c}}$ is the inner product of $\tilde{p}_{i}$ and $\tilde{p}_{\text {tot }}$. It follows that $d_{i}^{\mathrm{c}} / \sum_{j} d_{j}^{\mathrm{c}}$ represents the component of $\tilde{p}_{i}$ in the direction of $\tilde{p}_{\text {tot }}$.

\section{B. Properties of the Various Metrics}

Note that the first three metrics are non-negative and have a minimum equal to 0 when power delivery is constant. Therefore, a market participant with a very large variability is always penalized with a high power-regulation cost.

In contrast, the contribution metric can be either negative or positive, indicating whether individual market participants compensate or add to fluctuations in the total power profile. It might not penalize a participant, depending on whether the fluctuations of its power profile increase the total regulation cost or reduce it. In the latter case, this participant's powerregulation cost is negative, i.e., it is a revenue. Consequently, for the contribution metric, we can use one-price or two-price settlement schemes, like in the case of energy imbalances (in the latter case, any positive revenue would be made null). In the case-studies below, only one-price settlement will be considered. Overall, one can conclude that the first three metrics do not reflect the market participants' contribution to the overall imbalance, whereas the contribution metric (as suggested by its name) does so.

Let us examine the resilience of the four proposed metrics to collusion. Consider that a set $\mathcal{M}$ of market participants colludes and appears as a single aggregated market participant. 
Call $d_{\mathcal{M}}^{c}$ the contribution metric that is attributed to this aggregated participant in the new resulting market. Then

$$
d_{\mathcal{M}}^{\mathrm{c}}=\sum_{i \in \mathcal{M}} d_{i}^{\mathrm{c}}
$$

This follows immediately from (13) and from the fact that $\tilde{p}_{\text {tot }}(t)$ is not modified by the aggregation. In other words, the contribution metric is additive. It follows that the powerregulation costs are also additive, in particular

$$
R_{\mathcal{M}}^{p}=\sum_{i \in \mathcal{M}} R_{i}^{p}
$$

whenever the metric $d$ used in (9) is the contribution metric (here $R_{\mathcal{M}}^{p}$ is the power-regulation cost attributed to $\mathcal{M}$ ). It follows that the power-regulation costs attributed by using this metric are also additive.

In contrast, the additivity property does not hold for the first three metrics. We have, for the power capacity metric,

$$
d_{\mathcal{M}}^{\mathrm{pc}} \leq \sum_{i \in \mathcal{M}} d_{i}^{\mathrm{pc}}
$$

and the same holds for the integrated mismatch and the mileage metrics. This follows from the triangular inequality for $L_{p}$ norms. In most case, we expect the inequality in (16) to be strict for each of the first three metrics.

Consider now the case where the metric used for the computation of power-regulation costs is one of the first three metrics. Then we have

$$
R_{\mathcal{M}}^{p}=\frac{d_{\mathcal{M}}}{d_{\mathcal{M}}+\sum_{j \notin \mathcal{M}} d_{j}} c_{\text {tot }}
$$

where the notation $d_{\mathcal{M}}, d_{i}$ stands for $d_{\mathcal{M}}^{\mathrm{pc}}, d_{i}^{\mathrm{pc}}$ [resp. $d_{\mathcal{M}}^{\mathrm{im} k}$, $d_{i}^{\mathrm{im} k}$ or $\left.d_{\mathcal{M}}^{\mathrm{m}}, d_{i}^{\mathrm{m}}\right]$. Note that for $a>0$ the function $x \mapsto \frac{x}{x+a}$ is increasing.

Apply this to $x=d_{\mathcal{M}}, x^{\prime}=\sum_{i \in \mathcal{M}} d_{i}$ and $a=\sum_{j \notin \mathcal{M}} d_{j}$, it follows that

$$
R_{\mathcal{M}}^{p} \leq \sum_{i \in \mathcal{M}} R_{i}^{p}
$$

whenever the metric is one of the first three metrics, and in general the inequality in (18) is strict.

We conclude that the first three metrics are sub-additive, as are the power-regulation costs. It follows that there is an incentive for market participants to appear as an aggregate rather than as individuals. In contrast, with the contribution metric, the power-regulation cost attributed to any aggregate of participants is the sum of the individual power-regulation costs, and there is no such incentive.

In order to distribute the regulation cost (9) at the end of each market time-unit $\mathcal{T}$, each of the four metrics needs to be computed based on actual measurements. In practice, this can be done as follows. Each market participant samples her own power profile during a market time-unit $\mathcal{T}$ at $n$ time instants $t_{1}, \ldots, t_{n}$. At the end of the market time-unit, each market participant can compute her sampled energy-neutral power profile. Even if the sampling times $\left\{t_{k}\right\}_{k=1}^{n}$ do not perfectly coincide across market participants, the first three metrics can be well estimated locally based on this vector of measurements. The fourth metric however requires knowledge of the total sampled energy-neutral power profile. This "total profile" makes sense only if the market participants are synchronized and sample their profiles at the same time instants $\left\{t_{k}\right\}_{k=1}^{n}$. Subsequently all measurement vectors need to be communicated to a central collection point where their sum is computed and with it the contribution metric. Alternatively, the total energy-neutral profile can be itself measured at the same time instants and the resulting regulation cost distributed only among instrumented market participants.

In summary, among the four metrics, the contribution metric has highly desirable properties: it reflects market participants' contributions to the total imbalance and is resilient to collusion. In practice, however, its estimation is more complex than the first three metrics, as it requires synchronized measurements and induces higher communication costs. In the following we compare numerically the attribution of regulation costs according to the four metrics.

\section{Numerical Evaluation of the Proposed Metrics}

\section{A. System Model and Parameters}

We consider a simple market composed of strategic players $i=1, \ldots, n$ who are all wind producers, plus one nonstrategic player who represents the aggregate load. Our goal is to study the decisions of the strategic players for the next time-slot. After day-ahead market clearing, it is expected that player $i$ will deliver constant power $q_{i}^{f}$ for the entire duration of the time-slot. In real-time, at a given time $t$, producer $i$ can produce up to $q_{i}(t)$ units of power at time $t$ and his only strategic decision can be to curtail its production and produce $p_{i}(t) \leq q_{i}(t)$. We also assume that the value $q_{i}(t)$ is known to producer $i$, this corresponds to an ideal case of having access to perfect information. This value is equal to $q_{i}(t)=q_{i}^{f}+W_{i}(t)$ where $W_{i}(t)$ is the deviation from the forecast. In the numerical evaluation, the values $W_{i}(t)$ are generated by using a temporal covariance model inspired by the model in [13], where marginal predictive densities for every lead time have a beta distributions with mean $q_{i}^{f}$. To keep things simple, we consider that the load is not strategic (there is no player associated to the loads), is perfectly forecast, and is constant over the next time-slot. These are simplifying assumptions that can be readily relaxed. After market clearing, we expect perfect balance between generators' power production and load forecasts, thus the load power profile in the next time-slot is constant and equal to $-\sum_{i} q_{i}^{f}$.

1) Regulation Costs: The fraction of the power-regulation costs attributed to player $i$ is given by (8) and (9), with $\tilde{p}_{\text {tot }}(t)=\sum_{i=1}^{n} p_{i}(t)-\sum_{i} q_{i}^{f}$ and $d_{i}$ is the value for player $i$ of the chosen metric (one of the four metrics defined in Section IV). As the load is assumed to be constant over the time scale of interest, its metric is 0 and therefore does not bear any regulation costs in this simple example.

In order to complete the analysis, we need to also account for the energy-regulation costs; we take

$$
\pi^{\mathrm{b}}\left(E_{i}-E_{i}^{\mathrm{f}}\right)=\frac{E_{i}-E_{i}^{f}}{\sum_{i=1}^{n}\left(E_{i}-E_{i}^{f}\right)} R^{e}, \quad i=1, \ldots, n
$$


where $R^{e}$ is given by (8) with $E_{\text {tot }}=\sum_{i=1}^{n}\left(E_{i}-E_{i}^{f}\right)$. For the load the energy-regulation cost is 0 . The total regulation cost for player $i=1, \ldots, n$ is equal to the sum of (9) and (19).

For the numerical evaluation, we choose an instantaneous cost function $f$ equal to:

$$
f(x)=\alpha x+\beta x^{2} .
$$

We set $\beta=5$ and compare the results for $\alpha=-1$ and $\alpha=0$. The case $\alpha=-1$ represents the current frequent practice where production is encouraged (despite the penalty for deviating from the bid, each supplementary unit produced is still rewarded at a unit price of 1 - thus, a small overproduction is rewarded). The case $\alpha=0$ discourages both over-production and under-production.

2) Nash Equilibrium: When a strategic player $i$ decides to produce a profile $p_{i}$, it influences both its energy and power-regulation costs. Her actions also influence the cost that others have to pay, as the profile $p_{i}$ modifies $R^{e}$ and $R^{p}$. In the following, we will explore numerically a possible equilibrium situation in which no actor has an incentive to deviate unilaterally from her power profile.

For this, we consider the classic notion of Nash equilibrium [14] that, in our case, is defined as follows. Let $p_{-i}$ denote the power profiles of all players except $i$, for $i=1, \ldots, n$. A tuple of profiles $p_{1} \ldots p_{n}$ is a Nash equilibrium if for all player $i$,

- For all $t: 0 \leq p_{i}(t) \leq q_{i}(t)$;

- The profile $p_{i}$ minimizes the cost of player $i$, given by (9) and (19), given that the power profiles of the others, $p_{-i}$, are fixed.

In our case, the existence of a Nash equilibrium is guaranteed by the continuity of the cost function.

\section{B. Numerical Evaluation of the Nash Equilibria}

1) Numerical Method: We implemented a numerical algorithm that computes a Nash equilibrium for the four metrics. For each metric, we run our algorithm on the same forty scenarios for $q^{f}$ and $p^{f}$. Our numerical algorithm uses an iterative procedure that converges to a Nash equilibrium. For a player $i$, denote by $p_{i}^{*}\left(p_{-i}\right)$ the power profile that minimizes the cost of player $i$ given that the profiles of the other players $p_{-i}$ remain constant. Initially, we set the power profile of every player equal to $p_{i}(t)=q_{i}(t)$ (the amount of energy that would be produced without control, which is the maximum production for the wind producer). Then we repeatedly update the power profiles $\left(p_{i}\right)$ in a round-robin fashion, by setting $p_{i}=0.5 p_{i}+0.5 p_{i}^{*}\left(p_{-i}\right)$ until $\left|p_{i}-p_{i}^{*}\left(p_{-i}\right)\right| \leq 10^{-7}$. Convergence is reached after a maximum of a few hundreds of iterations in all tested cases.

2) Average Performance of Nash Equilibria: In Table I, we report the total regulation cost, averaged over the forty scenarios. We compare seven values:

- the total regulation costs given by the Nash equilibria (one for each metric),
TABLE I

AVERAGE COST OVER ALL SCENARIOS.

\begin{tabular}{|c|c|c|c|c|c|c|c|}
\hline \multicolumn{8}{|c|}{ Average cost when $\alpha=-1$ and $\beta=5$} \\
\hline & $d^{\mathrm{pc}}$ & $d^{\text {iml }}$ & $d^{\operatorname{im} 2}$ & $d^{\mathrm{m}}$ & $d^{\mathrm{C}}$ & no control & optimal \\
\hline Total cost & 5.75 & 5.64 & 5.54 & 6.16 & 5.47 & 6.83 & 5.33 \\
\hline Energy cost & 3.39 & 3.35 & 3.55 & 3.54 & 3.47 & 3.62 & 3.68 \\
\hline Power cost & 2.37 & 2.29 & 2.00 & 2.62 & 2.00 & 3.21 & 1.65 \\
\hline \multicolumn{8}{|c|}{ Average cost when $\alpha=0$ and $\beta=5$} \\
\hline & $d^{\mathrm{pc}}$ & $d^{\mathrm{im} 1}$ & $d^{\mathrm{im} 2}$ & $d^{\mathrm{m}}$ & $d^{\mathrm{c}}$ & no control & optimal \\
\hline Total cost & 3.08 & 3.09 & 3.17 & 3.52 & 3.07 & 7.33 & 2.99 \\
\hline Energy cost & 2.14 & 2.16 & 2.34 & 2.30 & 2.37 & 4.12 & 2.15 \\
\hline Power cost & 0.95 & 0.93 & 0.83 & 1.22 & 0.70 & 3.21 & 0.83 \\
\hline
\end{tabular}

- the total regulation costs when there is no control, i.e. each producer maximizes her production (she sets $p_{i}(t)=$ $\left.q_{i}(t)\right)$,

- the optimal, obtained from a hypothetical centrally computed allocation that minimizes $R^{e}(\bar{p})+R^{p}(\tilde{p})$ while respecting the constraints $p_{i}(t) \leq q_{i}(t)$ for every player $i$ and every time $t$. The optimal is not feasible in practice but serves as a benchmark.

We make two key observations. First, all metrics lead the producers to adopt power profiles that reduce the total regulation costs compared to producing the maximal power. These regulation costs are all strictly greater than the optimal allocation, meaning that no metric encourages players to adopt a globally optimal allocation. Second, the average total regulation cost of the Nash equilibrium with the contribution metric is lower than with all other metrics and is only a few percent off the optimal. Also, the average power-regulation costs with the contribution metric is always smaller than with the others. This can be explained by the fact that for the contribution metric, $d_{i}$ can be negative when a player helps the system, meaning that a player can be paid to help the system. For the other metrics, the quantity $d_{i}$ is always positive, hence even if a player can help the system, he always has an incentive to have a flat profile, if possible.

\section{3) Qualitative Difference between the Different Metrics:}

We now focus on one of the forty scenarios for which we plot the production as a function of time in Fig. 2 (for $\alpha=-1$ ) and Fig. 3 (for $\alpha=0$ ). These show the production of two players with different characteristics: Player 1 has a maximal production that varies over time whereas Player 2 has a constant maximal production (this occurs when the generator saturates at its maximum capacity). We compare the Nash equilibria when using the contribution metric with using the integrated mismatch metric with $k=2$. For the other metrics, the results are qualitatively similar to the integrated mismatch metric with $k=2$ and are not shown for lack of space.

We observe first that when $\alpha=-1$ (Fig. 2), i.e., when extra energy is bought back despite producers deviating from their bids, all players produce close to their maximal capacity. As a result, the total production fluctuates (Fig. 2(c)). When $\alpha=0$ (Fig. 3), i.e., when the regulation cost penalizes overproduction as well as underproduction, players are more flexible and produce profiles that are flatter. The total production is then almost constant (Fig. 3(c)). Second, with all metrics other than the contribution metric, the players are encouraged to have an 


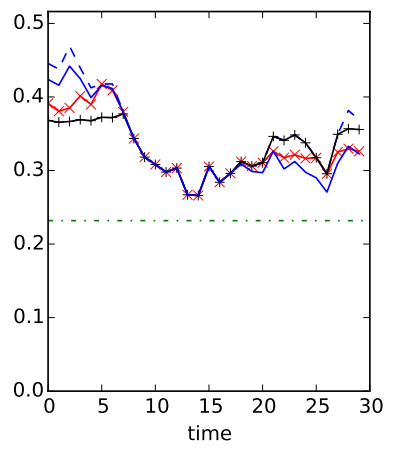

(a) Player 1

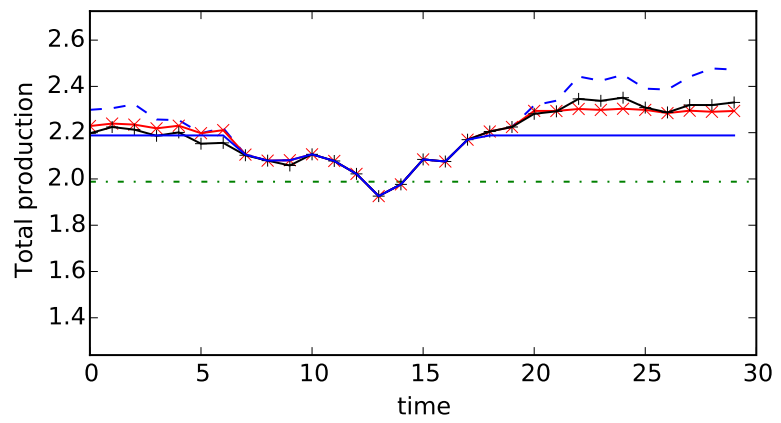

(c) Total production

Fig. 2. Production as a function of time in the Nash equilibrium when the instantaneous regulation cost is given by Equation (20) with $\alpha=-1$ and $\beta=$ 5 . For this scenario, the cost without control is -1.0 , the cost of the optimal allocation is 0.10 , of the equilibrium of the integrated mismatch metric -2.0 , and of the equilibrium of the contribution metric -2.5 .

almost constant profile (if possible). The situation is different with the contribution metric, because players are remunerated when they help the system. This is particularly visible in Fig. 3(a,b). This again explains why in Table $I$, the power costs, with the capacity and integrated mismatch metrics, are larger than with the contribution metric.

In summary, the evaluation in this section highlights the differences between the first three metrics and the contribution metric: This fourth metric encourages players to globally help the system, whereas the first three metrics encourage constant profiles. It also illustrates that there is little difference between the first three metrics.

\section{Vi. Application Results on a Country-wide Scale}

Owing to its current and projected penetration of renewable energy generation, Denmark is seen as an ideal test case area for analyzing how regulation costs that are induced by variability in power delivery would be redistributed to the actors of the system. We assume here that both wind power generation and demand may contribute to power fluctuations.

As a basis for simulations, power delivery profiles of wind power producers are generated using the CorWind simulation tool of the Technical University of Denmark, Department of Wind Energy, based on the spectral methods originally described in [15], [16]. This tool can generate realistic windspeed and power time-series over the entire Western Denmark area, accounting for the stochastic behavior of power fluctuations, and mimicking space-time covariance structures for

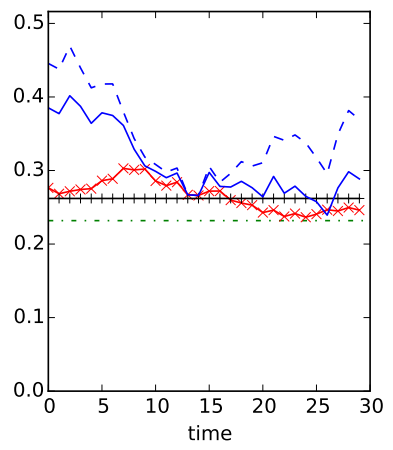

(a) Player 1

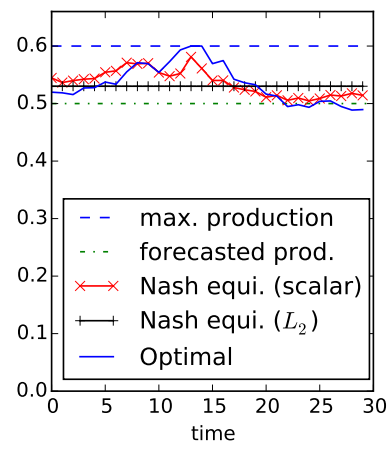

(b) Player 2

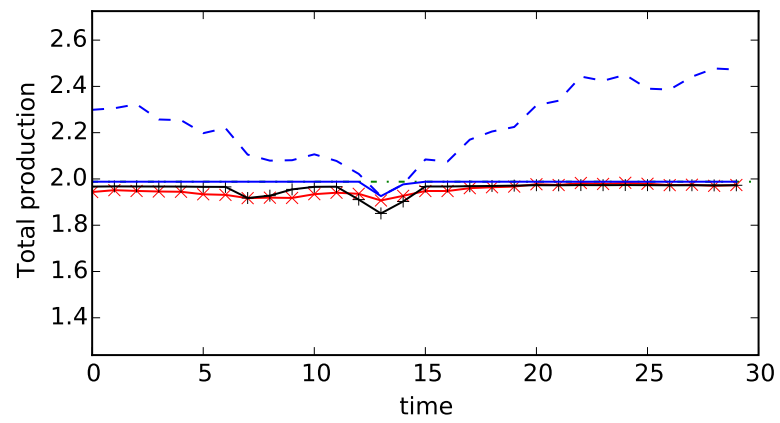

(c) Total production

Fig. 3. Production as a function of time in the Nash equilibrium when the instantaneous regulation cost is given by (20) with $\alpha=0$ and $\beta=5$. For this scenario, the cost without control is 6.5 , the cost of the optimal allocation is 0.10 , of the equilibrium of the integrated mismatch metric, 0.13 and of the equilibrium of the contribution metric 0.14 .

these fluctuations. The driving signal is given by meteorological simulations from the Weather Research and Forecasting (WRF) model. The conversion of wind speed to power is performed with simple steady-state power curves.

We consider here a setup with 41 wind farms located across Western Denmark and that respects the actual spatial density of wind farms. 7 were located offshore and 34 onshore. The total capacity is approximately $1.500 \mathrm{MW}$. An extensive description of the setup for this case-study, locations and capacities of wind farms, etc., can be found in [17]. In order to first illustrate the outcome of employing these attribution mechanisms, a random day of the year 2014 was chosen (March 1st), with a sampling based on a time resolution of 30 seconds for the power profiles from the wind farms. Based on powerdelivery profiles for the entire day, emphasis is placed on a given hour, 1-2pm, for illustrative purposes (Fig. 4). Realistic fluctuations for the power demand with the same resolution were also generated, in order to put in perspective power variability on the demand and supply sides. The stochasticprocess simulation-method employed, based on a first-order Gaussian process, can be found in [17].

Power-regulation costs and their attribution can be analyzed for several temporal resolutions. Currently the market timeunit in Denmark is 1 hour. However, with increased penetration of renewables, it is envisaged to shorten it to possibly 30 minutes, 15 minutes or even 5 minutes. These resolutions are then considered to calculate energy-neutral power profiles and the resulting power-regulation costs. An illustration of the 
impact of using different time-resolutions is provided in Fig. 4.

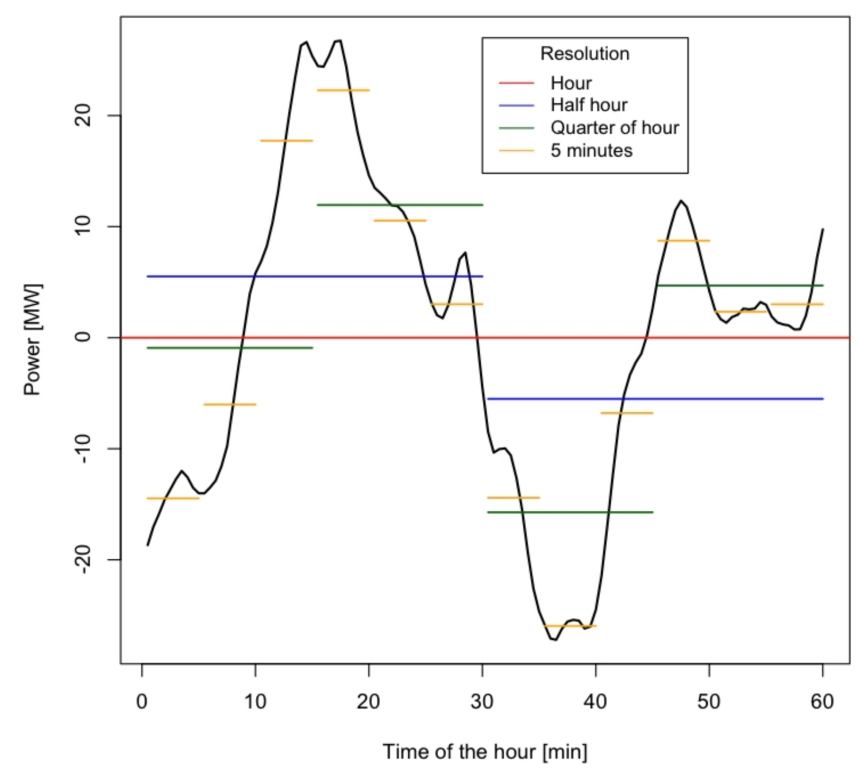

Fig. 4. From total power profiles to energy-neutral power profiles for the 1st of March, $1 \mathrm{pm}$ to $2 \mathrm{pm}$.

The maximum asolute value of these energy-neutral power profiles indicate how much capacity might be needed to perform power-regulation, hence to obtain the cost from activating reserves. Here, to determine those costs based on supply curves, we used sample supply curves from the German primary reserve market (Fig. 5), scaled to the size of the Danish reserve market. This is for the sake of illustration only, as the most important aspect of this analysis is how such costs will be distributed, not the level of the costs.

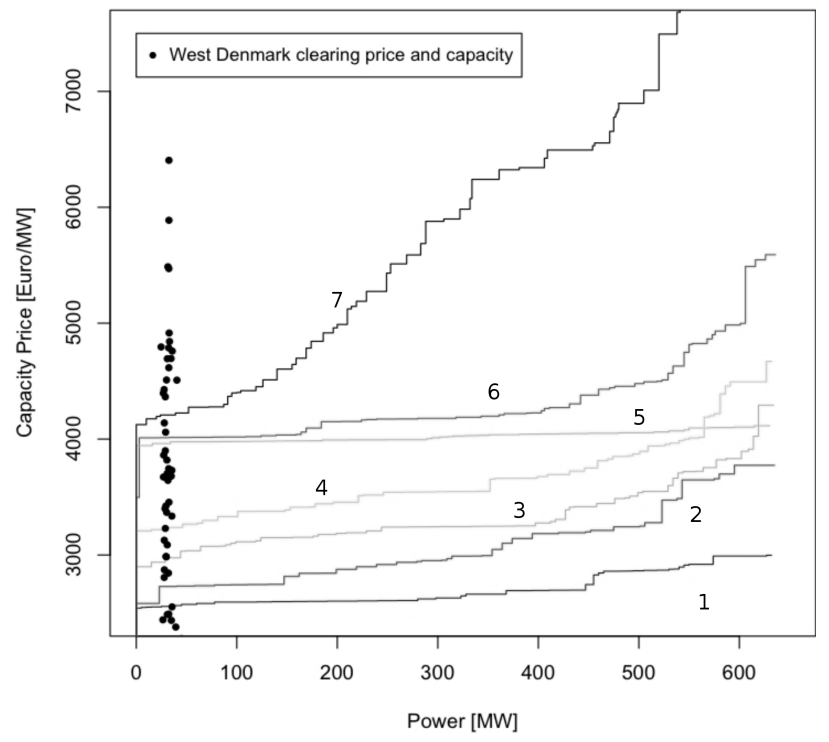

Fig. 5. Sample supply curves from the German primary reserve market, and scale difference with the Danish primary reserve clearing outcomes (from 2014).

The power-regulation costs induced by fluctuations in windpower delivery and consumption profiles were determined for the various temporal resolutions considered. The total costs for the procurement of primary reserves are summarized in Table II, for the various sample supply-curves shown in Fig. 5. A reduction in time resolution yields a decrease in overall power-regulation costs, because fluctuations have a lower magnitude. The order of magnitude of these costs also seems consistent with the real primary-reserve market hourlycosts in Western Denmark, which over the year 2014 had a mean value of $522 € / \mathrm{h}$ and a maximum value of $2.296 € / \mathrm{h}$. These costs, however, especially those calculated with the hour time-resolution, are likely to be overestimated because, even if market time-units are hourly in the Danish regulation markets, reserves are operated on finer time steps.

TABLE II

TOTAL POWER-REGULATION COSTS FOR 3 TIME RESOLUTIONS AND 7 SUPPLY CURVES.

\begin{tabular}{|l|r|r|r|}
\hline \hline Curve id. & $\begin{array}{l}\text { Hour } \\
\text { resolution } \\
{[€ / \mathrm{h}]}\end{array}$ & $\begin{array}{l}\text { Half hour } \\
\text { resolution } \\
{[€ / \mathrm{h}]}\end{array}$ & $\begin{array}{l}\text { Quarter } \\
\text { of hour } \\
\text { resolution } \\
{[€ / \mathrm{h}]}\end{array}$ \\
\hline \hline 1 & 436.64 & 365.78 & 275.28 \\
\hline 2 & 515.84 & 416.33 & 313.78 \\
\hline 3 & 532.52 & 443.46 & 395.18 \\
\hline 4 & 598.28 & 492.83 & 373.14 \\
\hline 5 & 655.12 & 550.95 & 417.84 \\
\hline 6 & 691.89 & 574.26 & 436.96 \\
\hline 7 & 1051.76 & 841.71 & 601.84 \\
\hline \hline
\end{tabular}

We computed the values of the 4 proposed metrics for all 41 wind power producers, as well as for the demand. We place emphasis on the case of 15-minute time resolution and the primary-reserve supply-curve no. 2 . The share of the total costs to be supported by each generator during that single hour is illustrated in Fig. 6. These results can be readily compared to the case where power-regulation costs would be socialized and fully covered by the demand side. The first row gives the respective contribution of each actor to the overall costs, depending on the metric; the second row gives the powerregulation costs per MWh generated. For the case of the first row, a fully socialized case would translate to all bars for the 41 wind-power producers being at 0 , whereas the last bar would be at $100 \%$. For the second row, all costs would be attributed to the demand and reflected by the right-most bar in the graph.

Results for the integrated mismatch metric with $k=2$ are not shown because they are very similar to the case $k=1$. For this example hour, the contribution for the offshore wind farms (the first actors on the left side of the histograms) is significantly higher than for the onshore ones. The last bar on the right is for the demand; it has the most substantial contribution to power-regulation needs. Contributions and individual costs are different, depending on the metrics used. The largest difference, however, is between the first 3 metrics (capacity, integrated mismatch, mileage) and the contribution one. This is because, with the contribution metric, contribution and costs may be both positive and negative, as certain actors contribute positively by having power fluctuations that lead to lower power-regulation needs. Although results for a single hour look quite extreme, we notice that if calculated over a month period (Fig 7) the differences in power-regulation costs 
for the various suppliers, as well as demand, are much less. These are, however, not equivalent to a fully-socialized view of supporting power-regulation costs (especially on the demandside only), as differences in the contribution of the various actors, and related payments, will remain.

The numerical results in this section confirm the findings of the previous section: The first three metrics have similar performances and penalize variability; in contrast, the fourth metric encourages some forms of variability ( when it has a positive global effect) and penalizes some others - and is neutral to the absence of variability.

\section{CONCLUSIONS}

Acknowledging the fact that increasing penetration for renewable energy sources, potentially combined with more proactive demand, will yield increased power fluctuations in modern power-systems, we have argued that new mechanisms could be designed for attributing the costs from variability in power delivery. We have shown that these would distribute power-regulation related costs in a way that represents contributions to overall costs. This could be an alternative to fully socializing these costs.

With this proposal, and as electricity markets are interconnected, it will be of utmost importance to see how such mechanisms would work in a multi-area setup. Similarly, as such a proposal is for a better distribution of costs induced by fluctuating production and demand, it should be evaluated whether this would facilitate the deployment of storage and demand response in support of renewable energy integration.

\section{ACKNOWLEDGMENTS}

The authors acknowledge their colleagues at DTU Wind Energy (namely Poul E. Sørensen and Petr Maule), who provided us access to CorWind for the simulation of wind power fluctuations over Western Denmark. Four anonymous reviewers and an editor are also to be acknowledged for their comments and suggestions, which contributed to improving the manuscript submitted originally.

\section{REFERENCES}

[1] Chu, S., Majumdar, A., "Opportunities and challenges for a sustainable energy future" Nature, vol. 488, pp. 294-303, 2012.

[2] O'Connell, N., Pinson, P., Madsen, H., O'Malley, M., "Benefits and challenges of electric demand response: A critical review," Renew. Sust. Energy Rev., vol. 39, pp. 686-699, 2014.

[3] Ela, E., Milligan, M., Kirby, B., "Operating reserves and variable generation - A comprehensive review of current strategies," NREL Tech. Rep., NREL/TP-5500-51978, 2011.

[4] Ela, E., Tuohy, A., Milligan, M., Kirby, B., Brooks, D., "Alternative approaches for incentivizing the frequency responsive reserve ancillary service," The Electricity Journal, vol. 25, no. 4, pp. 88-102, 2012.

[5] Ela, E., Gevorgian, V., Tuohy, A., Kirby, B., Milligan, M., O’Malley, M., "Market designs for the primary frequency response ancillary service Part I: motivation and design," IEEE T. Power Syst., vol. 29, no. 1, pp. 421-431, 2014.

[6] Hiroux, C., Saguan, M., "Large-scale wind power in European electricity markets: Time for revisiting support schemes and market designs?" Energy Policy, vol. 38, no. 7, pp. 3135-3145, 2010.

[7] Meyn, S., Negrete-Pincetic, M., Wang, G., Kowli, A., Shafieepoorfard, E., "The value of volatile resources in electricity markets," Proc. 49th IEEE Conference on Decision and Control (CDC), pp. 1029-1036, 2010.
[8] ENTSO-E (2014). ENTSO-E Overview of transmission tariffs in Europe: Synthesis 2014. Tech. Rep.: https://www.entsoe.eu/publications/ market-reports/Documents/SYNTHESIS_2014_Final_140703.pdf.

[9] Eyer, J., Corey, G., "Energy storage for the electricity grid: Benefits and market potential assessment guide," Tech. Rep., Sandia National Laboratories (pp. xvi and 102-104), 2010.

[10] Morales, J.M., Conejo, A., Madsen, H., Pinson, P., Zugno, M., "Integrating Renewables in Electricity Markets - Operational Problems”. New York, NY, USA: Springer, 2014.

[11] Dent, C., Bialeck, J., Hobbs, B., "Opportunity cost bidding by wind generators in forward markets: Analytical results," IEEE Trans. on Power Systems, vol. 26, no. 3, pp. 1600-1608, 2011.

[12] He, G., Chen, Q., Kang, C., Pinson, P., Xia, Q., "Optimal bidding strategy of battery storage in power markets considering performance based regulation and battery cycle life," IEEE Trans. on Smart Grid, available online, 2015.

[13] Pinson, P., Girard, R., "Evaluating the quality of scenarios of short-term wind power generation," Appl. Energy, vol. 96, pp. 12-20, 2012.

[14] Osborne, M.J., Rubinstein, A., "A Course in Game Theory". Boston, MA, USA: MIT Press, 1994

[15] Sørensen, P., Cutululis, N.A., Vigueras-Rodriguez, A., Madsen, H., Pinson, P., Jensen, L.E., Hjerrild, J., Donovan, M., "Modelling of power fluctuations from large offshore wind farms," Wind Energy, vol. 11, no. 1, pp. 29-43, 2008.

[16] Sørensen, P., Cutululis, N.A., "Wind farms' spatial distribution effect on power system reserves requirements," Proc. IEEE International Symposium on Industrial Electronics (ISIE), pp. 2505-2510, 2010.

[17] Bona, F., "Pricing variability in power delivery in markets with significant renewable energy generation," M.Sc. thesis, Technical University of Denmark \& Politecnico di Milano, 2015. Available online: pierrepinson. com/docs/Bona2015.pdf 

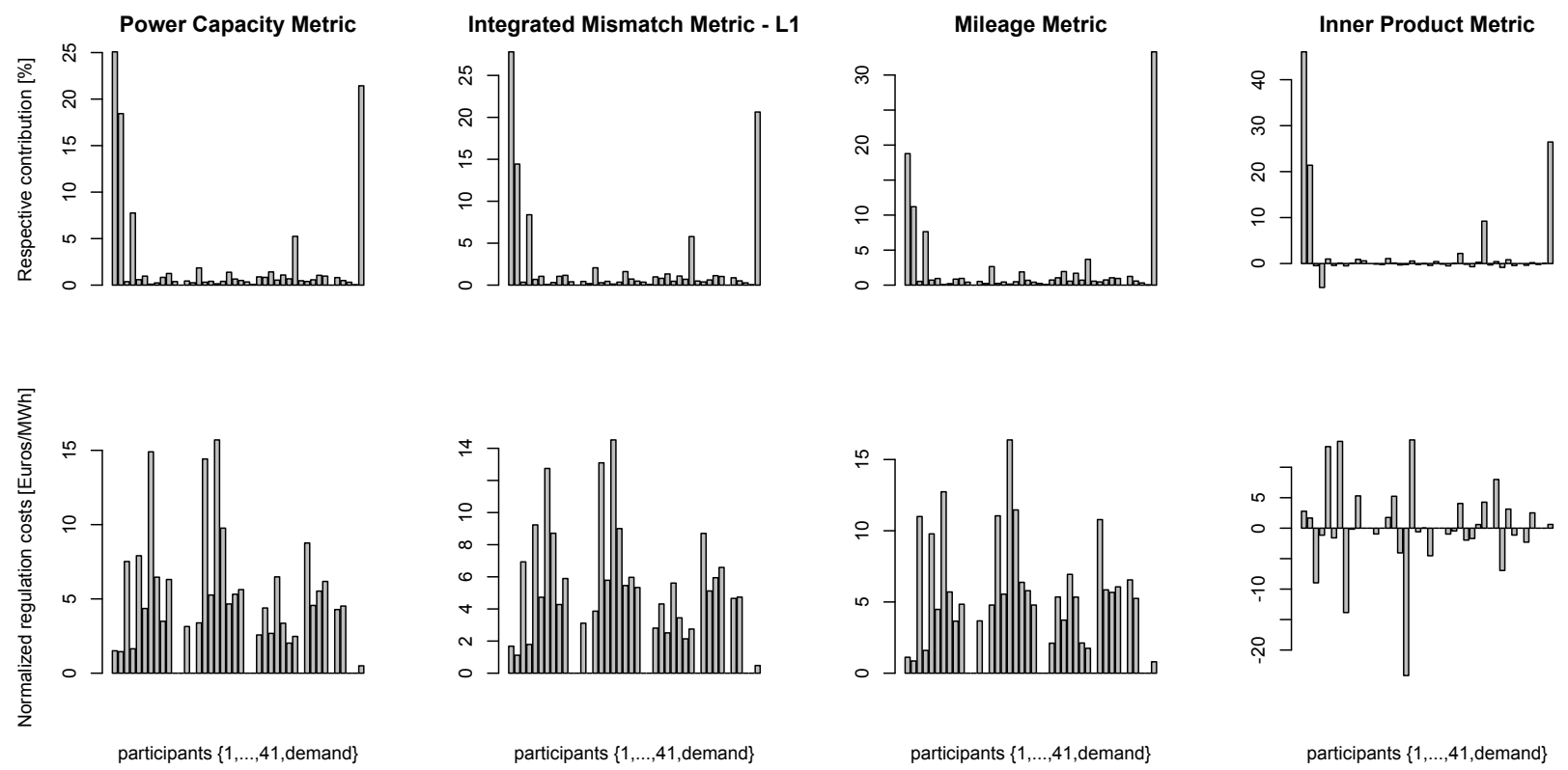

Fig. 6. Outcome of the attribution mechanisms for the 1st of March 2014, 1pm to 2pm, based on 15-min resolution.
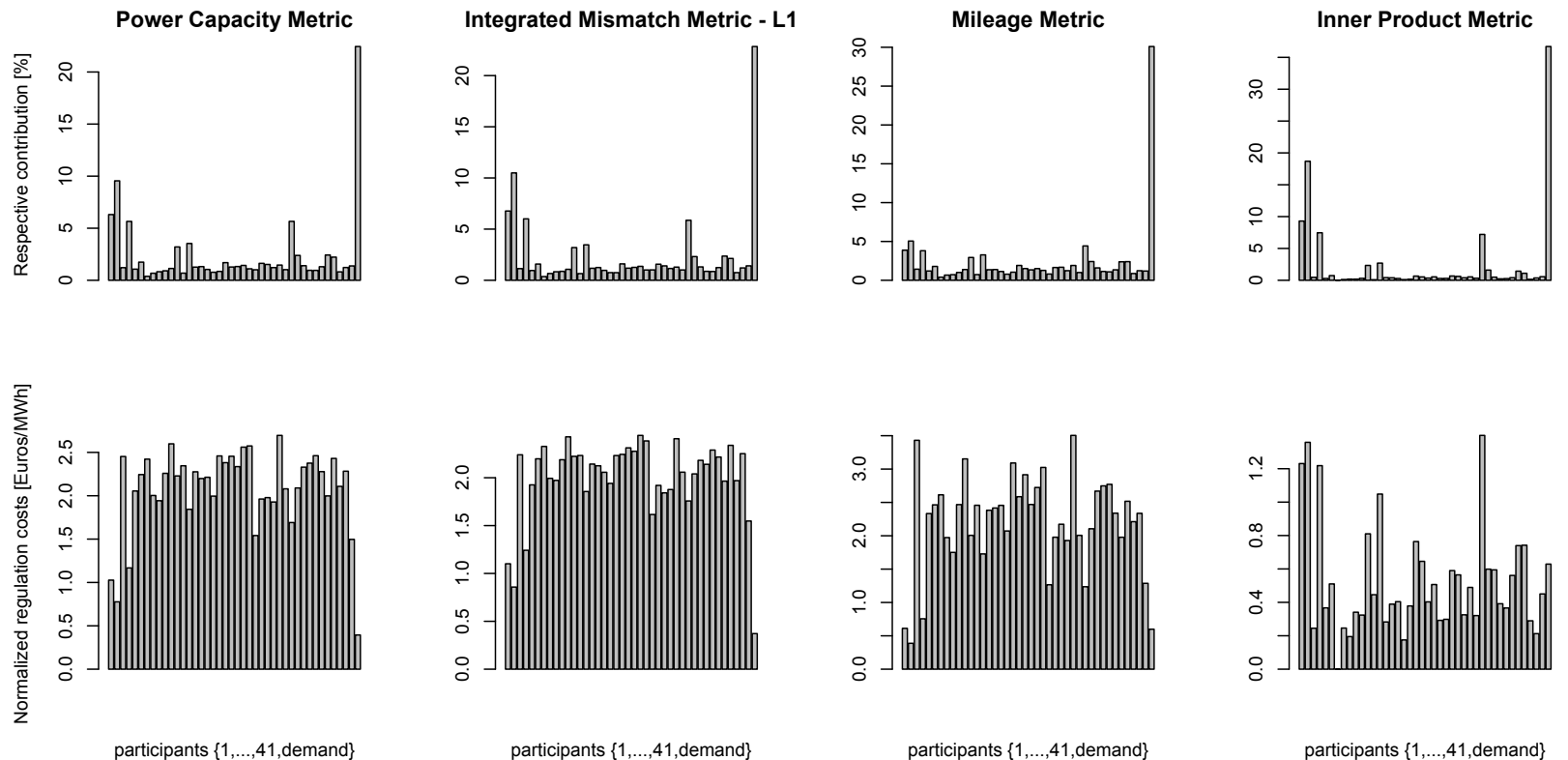

Fig. 7. Outcome of the attribution mechanisms for the entire month of March 2014, based on 15-min resolution. 